\title{
Perspectives on the Impact of the COVID-19 Pandemic on the Sports Medicine Surgeon: Implications for Current and Future Care
}

Kyle N. Kunze, MD, Peter D. Fabricant, MD, MPH, Robert G. Marx, MD, MSc, Benedict U. Nwachukwu, MD, MBA*

\section{KEYWORDS}

- SARS-Cov-2 • COVID-19 • Sports medicine • Athletes • Orthopedics

- Practice management

\section{KEY POINTS}

- The COVID-19 (Coronavirus disease 2019) pandemic has presented considerable challenges for orthopedic sports medicine surgeons and their patients, requiring rapid adjustment.

- For sports medicine surgeons, major challenges have included navigating telemedicine, personal and institutional financial losses, and psychosocial impacts from providing care.

- For patients and athletes, major challenges have included delayed return to sport, incomplete or limited rehabilitation, and anxiety associated with traveling to health care settings.

- Clinicians must take the lessons learned thus far and continue to apply them now and for the future as a new normal evolves that consists of treating patients with injuries previously treated with traditionally normal methods.

- The authors speculate that practices will continue to adopt telemedicine as a standard of care, and distancing and transmission precautions will remain in place for the foreseeable future.

\section{INTRODUCTION}

The impact of the COVID-19 (Coronavirus disease 2019) pandemic has been immense and far reaching. At the time of this writing, almost 5 months after the effects of COVID-19 began to be recognized in the United States, the number of new cases remains uncontrolled in certain regions. ${ }^{1}$ Coinciding with this pandemic have been numerous unforeseen effects, ranging from socioeconomic ramifications to significant 
changes in the ways in which clinicians routinely evaluate and treat patients. ${ }^{2-4}$ At this point, orthopedic surgeons, although returning to some degree of normalcy with regard to their practices, must be ready to permanently adapt the changes that this pandemic has imposed.

The subspecialty of sports medicine in particular has felt a significant impact. With essentially all sports, ranging from novice to professional levels, being canceled or postponed for the foreseeable future, it is difficult to conjecture what the future of sports participation will entail. ${ }^{5}$ Patients with musculoskeletal injuries treated by sports medicine surgeons may have treatment delayed because of preventive measures introduced by health care institutions and prioritization of nonelective cases. From both patient and sports medicine surgeon perspectives, these barriers may also create significant financial crises. For example, restrictions on participation in sports and on performing elective surgeries may leave institutions with financial deficits or on the brink of bankruptcy. ${ }^{4,6}$ Although many clinicians remain optimistic that these barriers will be gradually rescinded, it is only speculation as to whether and when health care practices will return to normal volumes and routines.

The COVID-19 pandemic has raised many questions that remain unanswered; however, the financial, psychosocial, and physical impacts are clear. This article highlights from both patient and physician perspectives the impact of the COVID-19 pandemic on sports medicine surgeons. These perspectives are reinforced using an evidencebased review of recent literature highlighting the various impacts of the pandemic. It is the primary aim of this article to enlighten both the academic community and general public as to how this pandemic has and continues to influence current sports medicine practice and thought, in addition to how it is poised to change future practice, perhaps permanently.

\section{IMPACT ON SPORTS MEDICINE SURGEONS}

The current health care landscape has transformed orthopedic sports medicine care. The global pandemic has required surgeons to cater to the musculoskeletal needs of their patients in a way that respects their overall health and mitigates the risk of COVID-19 transmission. Some of the ways that sports medicine surgeons and health care institutions have adapted to this landscape are highlighted here, along with a perspective on how this has affected care delivery.

\section{Telemedicine: Treating Physical Problems Through Virtual Means}

The use of telemedicine has been expanded to include a large portion of patient visits in order to respect the physical barriers instated by government and state officials such that the risk of COVID-19 transmission be minimized. ${ }^{7,8}$ Specifically, the barriers associated with the provision of virtual musculoskeletal care have been removed in the wake of the COVID-19 global pandemic. Insurers now reimburse for telemedicine and legislature, which allows the provision of virtual care, even going as far as to allow the practice of telemedicine across state lines for some encounters in some jurisdictions. A health care industry analysis by Bestsenny and colleagues ${ }^{9}$ suggested that in health care there had been a rapid adoption of telemedicine with high rates of both patient and provider satisfaction.

There are many benefits and challenges for sports medicine surgeons in engaging in virtual visits with patients. Major limitations to the use of telemedicine include technical difficulties resulting in lag or premature ending or disruption of a visit, concern for the quality of the encounter, and inability to perform a complete physical examination. ${ }^{10}$ These limitations may negatively influence the diagnostic value of a new or return 
patient encounter. However, there are significant benefits to these visits, including decreased risk of COVID-19 transmission, saved travel time for patients, and potentially lower rates of no-shows. ${ }^{11}$ A recent article by Tenforde and colleagues ${ }^{12}$ sought to describe the results from a quality improvement initiative during a rapid adoptive phase of telemedicine for outpatient sports and musculoskeletal physicians where surveys were completed by 119 patients and 14 physiatrists. The investigators reported that telemedicine was primarily used for follow-up visits (70.6\% of visits); patients rated their experience as excellent or very good between $91.6 \%$ and $95.0 \%$ of the time with regard to having concerns addressed, communication, treatment plan development, convenience, and satisfaction; the rate of no-show was only $2.7 \%$; and the key barrier identified for the visits was technical issues.

Although telemedicine continues to expand and the efficiency and technical quality of visits will likely to continue to improve, the in-person physical examination is impossible to replicate, because this is a skill that is cultured and developed throughout training. Tanaka and colleagues ${ }^{10}$ recently proposed a set of checklist items and systematic virtual examination techniques to help address these limitations. Their recommendations for a virtual checklist include (1) ensuring the camera is secured in a steady position; (2) numerous space and positioning guidelines for the knee, hip, shoulder, and elbow depending on the extremity that is the chief complaint; (3) lighting recommendations; (4) clothing recommendations depending on the extremity that is the chief complaint; and (5) ensuring that the location allows the patient to speak privately. For the systematic virtual examination, the investigators propose various maneuvers that patients can perform in conjunction with provider instruction in the comfort of their own homes, and provide approximate weights of household items that can be used during provocative strength tests. Because telemedicine is likely to remain common in the examination and evaluation of patients presenting for sports medicine evaluations, development of further examination maneuvers and new technology that will allow improved examinations will be imperative. Future directions may include standardized video instructions for patients, application of motion capture technology, and more reliance on diagnostic aids. Sports medicine surgeons will need to adapt their diagnostic algorithm in the virtual era in order to continue to provide the highest quality of care for their patients.

\section{Financial Implications}

High-volume orthopedic sports medicine centers have been forced to quickly absorb significant revenue losses that could not have been anticipated. Because these institutions were unprepared for such rapid financial losses, many are on the verge of bankruptcy and, for most, a large source of income (elective surgery) has been suddenly and dramatically curtailed. ${ }^{4,13}$ As a result of financial pressures, many institutions have instituted pay decreases, furlough policies, and even orthopedic staff downsizing. In order to respond to these financial pressures and better align themselves with their institutional plight, hospital-employed surgeons may find themselves accepting pay decreases, and private-practice surgeons may seek out governmental loans to weather the financial storm. The elimination of elective surgery and by proxy income for sports medicine surgeons may have forced some to seek out alternative sources of income, including increased call and locum positions. As sports medicine surgeons return to a new normal, there is likely to be immense pressure to recoup the missed volume. However, the reality is that financial losses in the current fiscal year will have to be amortized in upcoming years and may have implications for surgeon decision making and workload. 
The likely ripple effects of the current financial difficulties associated with COVID-19 are that financial disaster planning will become a part of institutional contingency planning. Specifically, institutions will pay increased attention to their cash flow, cash reserve, and maintaining a health account receivable. In addition, surgeons have become sensitized to the importance of having cash on hand to cover ongoing monthly expenditures in the absence of monthly income. Financial wisdom suggests 6 months of typical salary as appropriate liquidity.

\section{Psychosocial Perspectives: Effects of Off-Service Deployment}

With governmental and institutional focus on resuming patient care and returning to a new normal, the short-term and long-term effects of being immersed in a critical care environment may be overlooked. Many physicians were previously required to care for patients with COVID-19 or volunteered to do so, which has been shown to be a significant stressor and to impose a large psychological burden. ${ }^{14,15}$ A recent worldwide study of the impact of COVID-19 on spine surgeons revealed that family health concerns and anxiety were common and significant stressors, and that loss of income, clinical practice, and extent of surgical management varied widely. It is plausible, and likely, that these trends exist for sports medicine surgeons as well. Because COVID-19 disproportionality affects individuals of an older age, ${ }^{16-18}$ older sports medicine surgeons may be at risk of increased susceptibility and this too may have a psychological impact. Because sports medicine surgeons are required to be in hospital settings, this anxiety may be unavoidable and frequent. Such studies highlight the challenges experienced by orthopedic providers and the need for guidelines to be established for (1) psychosocial treatment of these providers, and (2) for anticipation of a second COVID-19 wave.

\section{Research Productivity and Expectations}

There has been a profound increase in research productivity during recent months, which coincides with diminishing clinical responsibilities. Furthermore, there is likely an academic "gold rush" to take advantage of the high rate of acceptance for articles pertaining to the COVID-19 pandemic. Gazendam and colleagues ${ }^{19}$ recently described this phenomenon as the infodemic of journal publication. This group performed a systematic literature search and found that, over the 13-week period since the initial documentation of COVID-19, a total of 1741 articles pertaining to COVID19 and severe acute respiratory syndrome-coronavirus-2 (SARS-CoV-2) were published in scientific journals, representing an exponential increase in academic productivity. This group also noted a short time from submission to publication, and a higher proportion of commentaries and opinion articles in journals with high impact factors.

Although a similar exploratory study has yet to be performed specifically for orthopedic research, similar trends are likely present. This trend would have benefits but also potentially negative consequences, if increased academic output is a function of sacrifices in quality. However, benefits of this trend include both personal and institutional gains in academic productivity and the potential to advance the field at a much more rapid pace. Institutions may seek to institute research infrastructures that give them the propensity to maintain the productivity observed during this era both now and in future years.

\section{IMPACT ON PATIENTS AND ATHLETES}

Although sports medicine surgeons have been greatly affected by the COVID-19 pandemic, challenges for their patients are also extensive and must be recognized. 
Such challenges include enduring musculoskeletal pain and dysfunction in order to minimize travel and potential COVID-19 exposure, anxiety in association with traveling to and being in hospital settings, and return to sport. Some of these challenges, and the way in which sports medicine surgeons may contribute to addressing these challenges, are discussed here.

\section{Anxiety, Pain, or Safety: Which Should Be Prioritized?}

Patients have expressed significant concerns in coming into a hospital setting, whether this is for clinical evaluation or a surgical procedure. Although hospitals are prioritizing minimization of the risk of COVID-19 transmission, for patients not routinely part of hospital settings, this can be an overwhelming and anxiety-provoking experience. ${ }^{20}$ In contrast, social isolation has been shown to be associated with increased anxiety and stress, ${ }^{21}$ and the additional effects of musculoskeletal injuries in combination with this remains unknown. Because the balance of patient care, financial incentives, and COVID-19 risk is delicate, it will be imperative to appropriately counsel patients regarding this anxiety. The authors recommend taking advantage of clinical encounters in the telemedicine setting to prime patients to their experience in the hospital and to emphasize the precautions set in place to minimize the risk of COVID-19 transmission and the need to abide by these regulations. This approach may address anxiety through guiding expectations, addressing pain through conservative or surgical care in the inpatient setting, and ensuring the safety of all in these settings.

\section{Returning to Sport, Eventually}

Because amateur sporting events have been classified as low-priority events in the sequence of returning to a new normal, it is uncertain as to when, and the extent to which, sports will resume. Because athletes represent a large proportion of patients evaluated and treated by sports medicine surgeons, and because athletes remain sidelined, this has implications for both patients and providers.

There remain many questions for athletes. These questions include, (1) whether athletes who have contracted COVID-19 will experience long-term effects that influence their health and subsequently their game performance (and if so, what those long-term diseases and consequences are); (2) for those unaffected but that remain sidelined, how potential deconditioning or delayed treatment of a musculoskeletal injury will influence their propensity for return to sport and their performance; and (3) what the future of organized sports will look like. At the time of this writing, some professional sports, such as golf and mixed martial arts, have experienced some success in reinstating regular events because a surge of COVID-19 cases has not been observed (this may be a function of these sports being individual based). Hockey and basketball are sports with smaller teams, which may make this effort slightly less challenging than sports with large teams, especially because these sports were in the postseason. In order to facilitate a safe return to sport, some of these larger team sports organizations have explored the so-called bubble concept; however, professional baseball has experienced early setbacks, and other large spectator sports such as football may also if COVID-19 transmission cannot be adequately controlled. These complications indicate how difficult it will be for amateur sports, including high school and collegiate athletics, to resume regular schedules before the introduction of a widely available vaccine. It is hoped that future protocols can improve safety for these professional athletes and the staff that surround them.

It will be imperative for sports medicine surgeons to safely guide athletes to returning to sport, regardless of when that is or what it looks like. It will be essential for sports medicine surgeons and those involved in the care of athletes to counsel athletes to 
safely return to sport. In this way, clinicians may help their athletes to avoid injuries associated with returning to sport, such as ruptures of the Achilles tendon or anterior cruciate ligament. Close attention must be paid to fatigue and signs of imminent injury, such as pain with activity, and the athletes must be guided accordingly. Although clinicians are passionate about the return of sports and hope that athletes can soon return to doing what they love, their number 1 priority must be to facilitate a safe return.

\section{The Impact of COVID-19 on Rehabilitation and Home Injury}

Another unique cohort of patients being affected by the COVID-19 pandemic are those who underwent a surgical procedure in the period before the pandemic. These patients who required extensive rehabilitation and physical therapy were unable to receive it in most cases. Prohibiting this essential aspect of surgical recovery may predispose such patients to suboptimal outcomes compared with their counterparts who were able to successfully complete physical therapy and rehabilitation. It is also likely that such patients will endure a longer recovery than their counterparts accordingly. Sports medicine surgeons and physical therapists will need to be conscientious of this impact and help these patients experience the best clinical and functional outcomes possible.

As many individuals continue to seek ways of maintaining fitness and staying healthy during the pandemic, a trend in injuries related to home exercise programs may be observed. These patients may develop overuse tendinopathies of the biceps and rotator cuffs if they are not accustomed to performing these exercises regularly. As such, sports medicine surgeons are likely to continue to observe an increase in these types of injuries as long as gyms and fitness centers remain closed.

\section{A TRANSIENT CHANGE OR NEW NORMAL?}

There are many challenges that have been faced and new issues that will continue to be encountered as sports medicine surgeons care for patients and athletes in the midst of the evolving pandemic. Despite these challenges, they will endeavor to continue to provide the highest level of care to their patients and to get them back to what they love to do, whether that is walking along the river or hitting a buzzer beater. There will continue to be injuries regardless of the infectious burden imparted by COVID-19, and therefore clinicians have an obligation to their patients to find innovative and effective ways to treat them both mentally and physically. The rapidly evolving nature of the pandemic may make this process more challenging, because recommendations and epidemiologic data are dynamic; however, it is of the utmost importance to take the lessons learned thus far and continue to apply them now and for the future as a new normal evolves in the treatment of patients with injuries previously treated with traditionally normal methods. The changes that may be required cannot be predicted with any certainty, but the authors speculate that practices will continue to adopt telemedicine as a standard of care and that distancing and transmission precautions will remain in place for the foreseeable future.

\section{DISCLOSURE}

The authors have nothing to disclose.

\section{REFERENCES}

1. World Health Organization. Coronavirus disease 2019 (COVID-19). Situation Report April 29, 2020. Available at: https://nam03.safelinks.protection.outlook. 
com/?url=https\%3A\%2F\%2Fwww.who.int\%2Fdocs\%2Fdefault-source\%

2Fcoronaviruse\%2Fsituation-reports\%2F20200429-sitrep-100-covid-19.pdf\%

3Fsfvrsn\%3Dbbfbf3d1_6\&data=02\%7C01\%7Cr.mayakrishnan\%40elsevier.com

\%7C70ae398d1ef94726d77808d8566ccaac\%

7C9274ee3f94254109a27f9fb15c10675d\%7C0\%7C0\%

7C637354373097911192\&sdata=baLhM3c\%2FTL6Xpl4guVi5F3g\%

2BLOp1JsInS3plsnpgRiw\%3D\&reserved=0. Accessed July 30, 2020.

2. Khalatbari-Soltani S, Cumming RC, Delpierre C, et al. Importance of collecting data on socioeconomic determinants from the early stage of the COVID-19 outbreak onwards. J Epidemiol Community Health 2020;74(8):620-3.

3. Nicola M, Alsafi Z, Sohrabi C, et al. The socio-economic implications of the coronavirus pandemic (COVID-19): A review. Int J Surg 2020;78:185-93.

4. Vaccaro AR, Getz CL, Cohen BE, et al. Practice management during the COVID19 pandemic. J Am Acad Orthop Surg 2020;28(11):464-70.

5. Schellhorn P, Klingel K, Burgstahler C. Return to sports after COVID-19 infection. Eur Heart J 2020. https://doi.org/10.1093/eurheartj/ehaa448.

6. Khullar D, Bond AM, Schpero WL. COVID-19 and the financial health of US hospitals. JAMA 2020. https://doi.org/10.1001/jama.2020.6269.

7. Hollander JE, Carr BG. Virtually perfect? telemedicine for covid-19. N Engl J Med 2020;382(18):1679-81.

8. Loeb AE, Rao SS, Ficke JR, et al. Departmental experience and lessons learned with accelerated introduction of telemedicine during the COVID-19 crisis. J Am Acad Orthop Surg 2020;28(11):e469-76.

9. Bestsenny O, Gilbert G, Harris A, et al. Telehealth: a quarter-trillion-dollar postCOVID-19 reality? McKinsey \& Company; 2020. Available at: https://nam03. safelinks. protection. outlook.com/?url=https:\%2F\%2Fwww. mckinsey.com\%2F \% 2Fmedia\%2FMcKinsey\%2FIndustries\%2FHealthcare\%2520Systems\%2520and\% 2520Services\%2FOur\%2520Insights\%2FTelehealth\%2520A\%2520quarter\% 2520trillion\%2520dollar\%2520post\%2520COVID\%252019\%2520reality\% 2FTelehealth-A-quarter-trilliondollar-post-COVID-19-reality.pdf\&data=02\%7C01\% 7Cr.mayakrishnan\%40elsevier.com\%7C70ae398d1ef94726d77808d8566ccaac\% 7C9274ee3f94254109a27f9fb15c10675d\%7C0\%7C0\%

7C637354373097911192\&sdata=a4sen1492tt\% 2B92rS2dcSwW8oup7ElenAd83LaoY9dqM\%3D\&reserved=0. Accessed July 30, 2020.

10. Tanaka MJ, Oh LS, Martin SD, et al. Telemedicine in the era of COVID-19: the virtual orthopaedic examination. J Bone Joint Surg Am 2020;102(12):e57.

11. Atanda A, Pelton M, Fabricant PD, et al. Telemedicine utilisation in a paediatric sports medicine practice: decreased cost and wait times with increased satisfaction. J ISAKOS 2018;3(2):94.

12. Tenforde AS, laccarino MA, Borgstrom $\mathrm{H}$, et al. Telemedicine During COVID-19 for outpatient sports and musculoskeletal medicine physicians. PM R 2020. https://doi.org/10.1002/pmrj.12422.

13. Gilat R, Cole BJ. COVID-19, Medicine, and Sports. Arthrosc Sports Med Rehabil 2020;2(3):e175-6.

14. Elbay RY, Kurtulmus A, Arpacioglu S, et al. Depression, anxiety, stress levels of physicians and associated factors in Covid-19 pandemics. Psychiatry Res 2020;290:113130.

15. Lai J, Ma S, Wang Y, et al. Factors associated with mental health outcomes among health care workers exposed to coronavirus disease 2019. JAMA Netw Open 2020;3(3):e203976. 
16. Imam Z, Odish F, Gill I, et al. Older age and comorbidity are independent mortality predictors in a large cohort of 1305 COVID-19 patients in Michigan, United States. J Intern Med 2020. https://doi.org/10.1111/joim.13119.

17. Lloyd-Sherlock PG, Kalache A, McKee M, et al. WHO must prioritise the needs of older people in its response to the covid-19 pandemic. BMJ 2020;368:m1164.

18. Mueller AL, McNamara MS, Sinclair DA. Why does COVID-19 disproportionately affect older people? Aging (Albany NY) 2020;12(10):9959-81.

19. Gazendam A, Ekhtiari S, Wong E, et al. The "infodemic" of journal publication associated with the novel coronavirus disease. J Bone Joint Surg Am 2020; 102(13):e64.

20. Vindegaard N, Benros ME. COVID-19 pandemic and mental health consequences: Systematic review of the current evidence. Brain Behav Immun 2020. https://doi.org/10.1016/j.bbi.2020.05.048.

21. Xiao H, Zhang $Y$, Kong D, et al. Social capital and sleep quality in individuals who self-isolated for 14 days during the coronavirus disease 2019 (COVID-19) Outbreak in January 2020 in China. Med Sci Monit 2020;26:e923921. 